\title{
Turbulence via information field dynamics
}

\author{
Torsten A. Enßlin \\ Max Planck Institute for Astrophysics \\ Karl-Schwarzschild Str. 1, 85741 Garching, Germany \\ email: ensslin@mpa-garching.mpg.de
}

\begin{abstract}
Turbulent flows exhibit scale-free regimes, for which information on the statistical properties of the dynamics exists for many length-scales. The simulation of turbulent systems can benefit from the inclusion of such information on sub-grid process. How can statistical information about the flow on small scales be optimally incorporated into simulation schemes? Information field dynamics (IFD) is a novel information theoretical framework to design schemes that exploit such statistical knowledge on sub-grid flow fluctuations.
\end{abstract}

Keywords. Information theory, field theory, simulations, turbulence

IFD is a theoretical framework to construct information optimal field simulation schemes (Enßlin 2013). IFD permits the information fusion of the dynamical equations, statistical properties, and relevant observational data of a system. This is performed via information field theory (IFT, Enßlin 2009) and a maximum entropy-based optimal coding. The concept of IFD is illustrated below.

Data in computer memory is regarded as resulting from a field measurement process (bottom left). The ensemble of field states consistent with this data and other information can be reconstructed from this with the help of IFT (top left). The time evolution operator of the field $\mathrm{dy}$ namics produces the time evolved field ensemble (top right). This is then represented by data in computer memory via entropic matching of the two distribution functions (bottom right). The transformation rule relating initial and updated data is then the desired simulation scheme (bottom arrow), which incorporates the

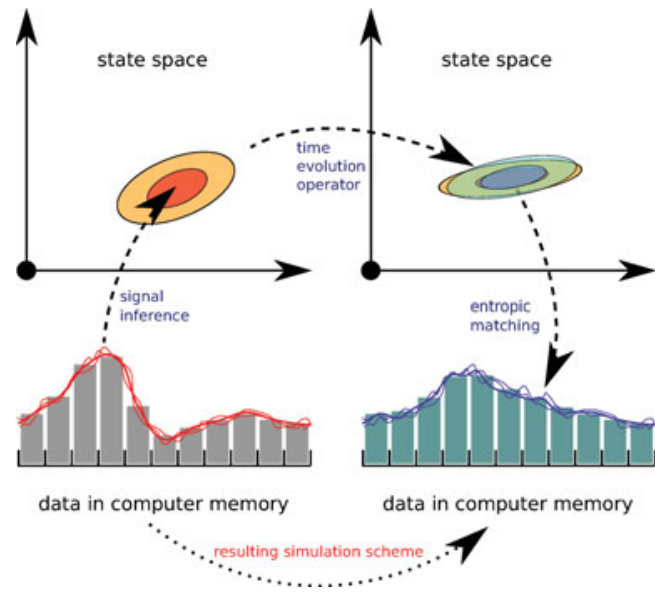
continuous field dynamics and available statistical knowledge information-optimally.

The operability of the IFD scheme has been demonstrated for a thermally excited Klein-Gordon field (Enßlin 2013) as well as for stochastic network dynamics (Ramalho et al. 2013). Application to hydrodynamics and plasma physics are in preparation.

\section{References}

Enßlin, T. A., Frommert, M., \& Kitaura, F. S. 2009, Phys. Rev. D 80, 105005

Enßlin, T. A. 2013, Phys. Rev. E 87, 013308

Ramalho, T., Selig, M., Gerland, U., \& Enßlin, T. A. 2013, Phys. Rev. E 87, 022719 